\title{
Extrusion Pressure Estimation in Axisymmetric Paste Extrusion ${ }^{\dagger}$
}

\author{
D. J. Horrobin \\ University of Melbourne, Australia* \\ and R.M. Nedderman \\ University of Cambridge, UK ${ }^{* *}$
}

\begin{abstract}
Paste extrusion is an important process used for manufacturing products based on particulate materials. From a theoretical viewpoint, paste extrusion resembles solid metal extrusion, for which extensive theory has been developed. In particular, exact solutions are available for many extrusion processes involving plane strain deformations, allowing extrusion pressures to be accurately determined in these situations. However, axisymmetric extrusion is more common in a paste flow context, and in this case simple alternative methods for estimating extrusion pressures are desirable.

In this article, we describe how exact solutions for plane strain extrusion can be applied empirically to axisymmetric geometries, to provide accurate estimates for extrusion pressures. The nominal extrusion pressures for plane strain and axisymmetric extrusions with equal area reductions appear to be similar, for both smooth-and rough-walled square-entry dies. The axisymmetric extrusion pressure can be estimated directly in the smooth wall case, while for rough walls, the contribution due to rigid sliding, away from the deforming zone, must also be considered.

Some new correlations describing combined results from slipline field solutions for plane strain extrusion are also presented.
\end{abstract}

\section{Introduction}

Paste extrusion is a common method for manufacturing objects from particulate materials. The process involves liquid addition to a powder to produce a deformable soft solid paste, which then undergoes extrusion to create the desired cross-sectional shape. This is typically followed by further processing, which might involve liquid removal to obtain a hard final product. Many materials not derived from dry powders, notably various foodstuffs, are also processed in a soft solid state, and so soft solid extrusion has a wider significance.

Typical pastes are dense, solid-liquid mixtures that exhibit yield behaviour when subjected to deforming loads, i.e. deformation does not begin (at least at an appreciable rate) until the load has reached a critical magnitude. The yield stress is found to be largely independent of hydrostatic pressure [1], and in many cases the stress in a deforming sample is only weakly dependent on the deformation rate and accumulated strain. In this sense, pastes resemble solid metals, both materials being perfectly plastic to a first approximation.

\footnotetext{
* Department of Mathematics and Statics, Victoria, 3010, Australia

** Department of Chemical Engineering, Pembroke Street, Cambridge, CB2 3RA, UK

† Received: May 21, 1999
}

For plane strain extrusion, the slipline field method allows extrusion pressures to be accurately determined for perfectly plastic materials, and plane strain slipline field results are often tabulated in standard metalforming texts [e.g. 2]. The plane strain geometry can be visualised from Figure 1 by imagining the wall to extend for a large distance perpendicular to the page. However, axisymmetric extrusion, where the entering and exiting flows have concentric circular cross-sections, is rather more common in paste processing.

The slipline field approach is difficult to apply to axisymmetric geometries, and so to obtain accurate solutions, numerical methods must be employed. In particular, the finite element method has been widely used for analysing extrusion processes. Numerical calculations can be costly and time-consuming, however, and simple methods for estimating forming loads are thus desirable. Extrusion pressures can be estimated, for both plane strain and axisymmetric deformations, using the limit analysis (upper and lower bound theorem) approach. Although these estimates are very accurate for plane strain extrusion, comparisons with finite element results have indicated that this is not generally the case for the axisymmetric geometry [3].

In this article, we briefly describe the perfect plasticity model, and some slipline field solutions for plane strain extrusion through square-entry dies. The upper 
Dound approach is also illustrated by some plane strain examples. We then show how plane strain slipline field results can be applied to axisymmetric extrusion, to produce better agreement with finite element extrusion pressures than is obtained from axisymmetric upper bound solutions. We shall consider problems involving Tresca boundary conditions, where the wall shear stress has a constant value where sliding occurs. If this value is zero, the walls are said to be perfectly smooth, and if it is equal to the material's shear yield stress, they are said to be perfectly rough. We shall only consider these two limiting cases, but the conclusions are expected to hold for intermediate, partly rough walls.

As a side issue, we also present some new correlations (equations 1, 7 and 9) describing combined slipline field results for plane strain extrusion through square-entry dies.

\section{Perfectly plastic materials}

As with other problems in continuum mechanics, the flow of a perfectly plastic material is described by a set of force equilibrium equations (body forces usually being neglected) and a set of constitutive relationships, which in this case comprise a yield criterion and a flow rule. The yield criterion determines the deviatoric stress magnitude at which deformation can occur. This critical stress cannot be exceeded, and below it the material is rigid. For a von Mises material, the appropriate measure of the stress magnitude is the sum of the squares of the deviatoric stress components.

The flow rule determines the ratios of the strain rate components, and for a von Mises material obeying the associated flow rule, these are equal to the

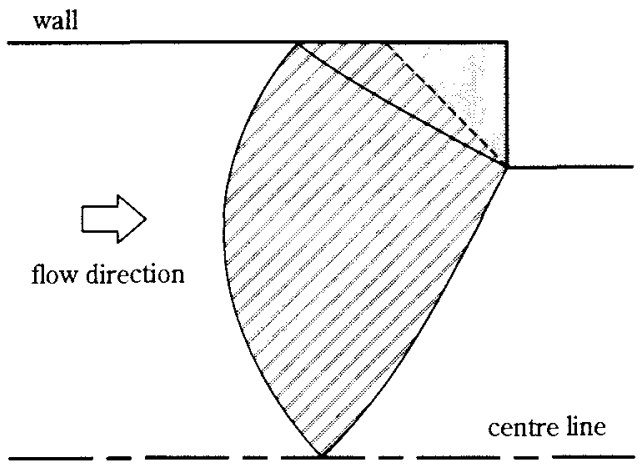

(a) Small reductions (illustrated for $\gamma=0.3$ ) ratios of the corresponding deviatoric stress components. Consequently, the deformation is volume conserving, i.e. the material is incompressible. The magnitudes of the strain rate components are independent of the stress components, and are determined by the kinematic boundary conditions, in this case the ram velocity. The extrusion pressure is therefore independent of extrusion rate.

\section{Slipline field solutions}

Under plane strain conditions, the velocity and strain rate in a given direction are both zero, and it is possible to obtain exact solutions for many problems using the slipline field method. This is an application of the method of characteristics, and is described in standard metal-forming texts [e.g. 2, 4, 5]; its details will not be discussed here. A number of slipline field solutions have been presented for extrusion with Tresca boundary conditions. The flow patterns are characterised by well-defined deforming zones separating the rigid material entering and exiting the die entry region. Depending on geometry and boundary conditions, there may also be a well-defined static zone on the die face, and surfaces within the deforming zone across which the velocity is discontinuous. These features can all be seen in the solutions described below.

Figure 1 shows the deforming zones, and associated static zones and velocity discontinuities, for plane strain extrusion through perfectly smooth, square-entry dies with two different fractional thickness reductions (denoted by $r$ ). Figure 1 (a) corresponds to a solution due to Hill [6] for extrusion through dies with small thickness reductions, the maximum reduction for which this solution is valid

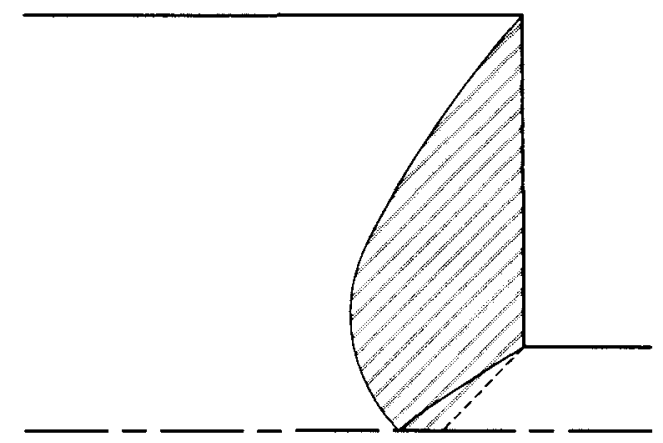

(b) Large reductions (illustrated for $r=0.8$ )

Fig. 1 Deforming zones, static zones and velocity discontinuities for plane strain extrusion through smooth, squareentry dies; from slipline field solutions. 
beipg $r=1 / 2$. Figure 1(b) corresponds to a solution due to Johnson [7] for extrusion through dies with large thickness reductions, the minimum reduction for which this solution is valid being $r=2 / 3$. Two solutions covering the range $1 / 2<r<2 / 3$ have been given by Green [8].

The extrusion pressures derived from these solutions are tabulated in standard texts [2] or can be calculated relatively quickly using a computer. (The solutions described in this article were all calculated on ' a PC using the Mathematica package.) These extrusion pressures are plotted in Figure 2 against the logarithm of the thickness ratio (entry thickness divided by exit thickness). They are made dimensionless by dividing by $\sqrt{3} k$, where $k$ is the material's shear yield stress; $\sqrt{3} k$ is equal to the uniaxial yield stress for a von Mises material. The results are found to lie approximately on two parabolic arcs with equations

$$
\begin{array}{rlrl}
\frac{P}{\sqrt{3} k} & =0.520(\sqrt{20.2 \xi+1}-1) & & r \leq 0.75 \\
& =0.0468 \xi^{2}+0.845 \xi+1.02 & r \geq 0.75
\end{array}
$$

where $\xi=\ln |1 /(1-r)|$.

\section{Plane strain upper bound solutions}

Extrusion pressures can be estimated for both plane strain and axisymmetric extrusion using the upper bound approach. An upper bound solution comprises a velocity field that satisfies the incompressibility constraint and the velocity boundary conditions for the problem. The rate of energy dissipation associated with this velocity field can be calculated without detailed knowledge of the stress field, as the flow rule and yield criterion uniquely determine the energy dissipation rate per unit volume at each point. The extrusion pressure is then obtained from an energy balance, and the upper bound theorem, due to Hill [9], states that this extrusion pressure is greater than or equal to the exact extrusion pressure. In other words, the material adopts the flow pattern that minimises the forming load. By examining different velocity fields, that with the lowest energy dissipation rate can be selected, and a close approximation to the exact extrusion pressure hopefully obtained.

Simple upper bound solutions for plane strain extrusion can be constructed by dividing the deforming zone into triangular sub-regions, within which the material moves rigidly. The incompressibility constraint requires that the velocity component normal to

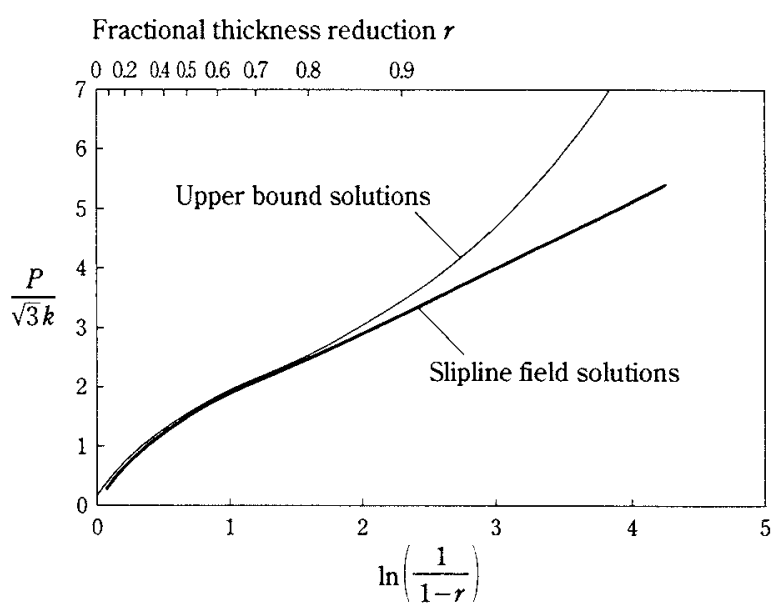

Fig. 2 Extrusion pressures for plane strain extrusion through smooth, square-entry dies.

an interface between sub-regions must be continuous, although the tangential velocity component can be different on either side. The interfaces are therefore velocity discontinuities. Energy dissipation only takes place at the discontinuities, and at external boundaries if the wall shear stress is non-zero.

Two such upper bound solutions, due to Kudo [10, 11], are shown in Figure 3. In each case, the length parameter $a$ is adjustable, and its value can be selected to give the lowest extrusion pressure. Similarly, the angles in the triangular sub-regions can be optimised, the most suitable triangular shapes being indicated in the figure. The velocity field in Figure 3(a) produces a lower extrusion pressure when the reduction is small (less than 60.2\%), and the optimum value for $a$ is

$$
a=4 \sqrt{\frac{r(1-r)}{7}}
$$

The resulting extrusion pressure is

$$
\frac{P}{\sqrt{3} k}=\sqrt{\frac{7 r}{3(1-r)}}
$$

The velocity field in Figure 3(b) produces a lower extrusion pressure for larger reductions, with $a$ and $P$ given by

$$
\begin{aligned}
& a=\sqrt{r(1-r)} \\
& \frac{P}{\sqrt{3} k}=\frac{16-9 r}{4} \sqrt{\frac{r}{3(1-r)}}
\end{aligned}
$$

Equations (3) and (5) are also plotted in Figure 2, where it is seen that the upper bound solutions provide very good estimates for the extrusion pressure over a wide reduction range, specifically for reductions less than about $85 \%$. At larger reductions, the upper bound and slipline field results diverge, although 


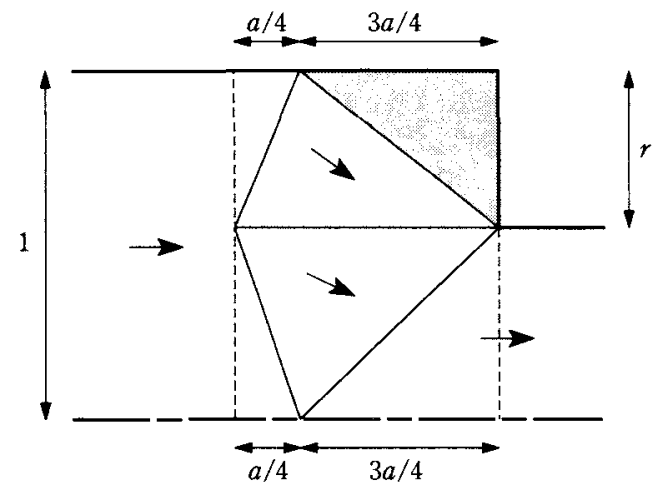

(a) Velocity field for $r \leq 60.2 \%$

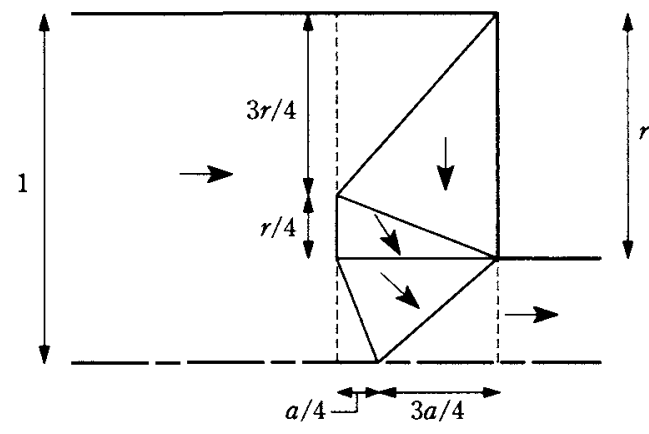

(b) Velocity field for $r \geq 60.2 \%$

Fig. 3 Velocity fields used in upper bound solutions for plane strain extrusion through smooth, square-entry dies.

it should be pointed out that the solution depicted in Figure 3(b) does not represent the best available upper bound solution in this region. Simple modifications to this solution result in lower extrusion pressure estimates at very large reductions, although agreement is still not as close as for reductions less than $85 \%$.

\section{Axisymmetric extrusion}

The slipline field method is not easily applied to axisymmetric extrusion, but accurate solutions to the equations of motion can still be obtained, at higher computational cost, by performing numerical calculations. For example, in a paste extrusion context, references [3] and [12] present some finite element results for extrusion of a perfectly plastic material through dies with various geometries and boundary conditions. The finite element extrusion pressures plotted in Figure 4, for axisymmetric extrusion through smooth, square-entry dies with diameter reductions between 4 and $92 \%$, are taken from these sources. The values are made dimensionless by dividing by the material's uniaxial yield stress $\sqrt{3} k$ and are plotted against $\xi=\ln |1 /(1-r)|$, where $r$ is again the linear reduction (in this case the fractional diameter reduction). The

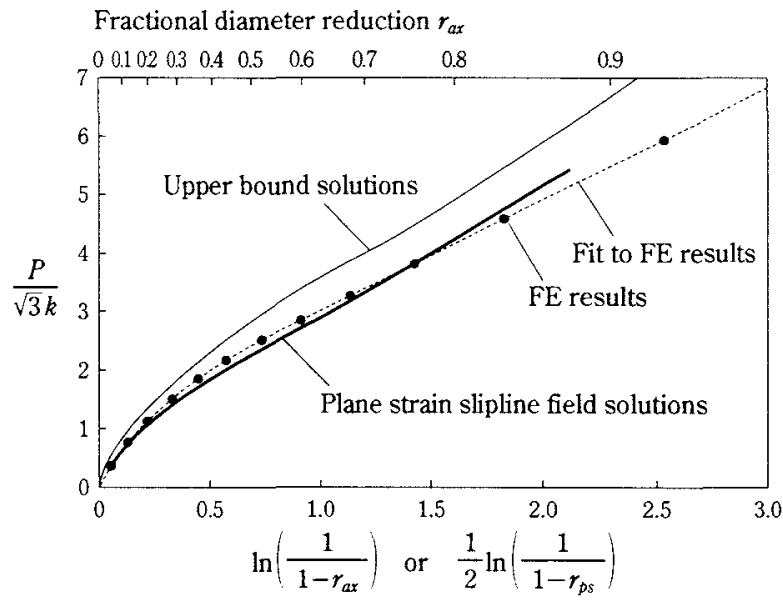

Fig. 4 Extrusion pressures for axisymmetric extrusion through smooth, square-entry dies.

dotted line in the figure is an empirical fit to the finite element data given by

$$
\begin{array}{rlrl}
\frac{P}{\sqrt{3} k} & =0.700(\sqrt{26.8 \xi+1}-1) & & r \leq 0.60 \\
& =1.92 \xi+1.08 & r \geq 0.60
\end{array}
$$

Since finite element analyses can be costly and time-consuming, it is desirable to find alternative procedures for determining forming loads with sufficient accuracy. Upper bound solutions exist for axisymmetric extrusion, but in this case it is not possible to construct deformation modes based on (axisymmetric) rigid blocks, due to the need to accommodate circumferential straining. The velocity fields employed therefore comprise deforming sub-regions, again separated by velocity discontinuities. As a result, calculation of the rate of energy dissipation is more complicated, and expressions for the extrusion pressure are generally more cumbersome than for plane strain extrusion $[3,12]$.

Among the better upper bound solutions for axisymmetric extrusion through square-entry dies are those due to Kudo [13]. These were developed before numerical solutions for extrusion problems were available, in the belief that they might provide accurate estimates for the extrusion pressure, over a wide reduction range, as in the plane strain case. The solutions will not be discussed in detail here (the details can be found in reference 12), but give rise to the upper bound curve shown in Figure 4 . Comparison with the finite element results indicates that the axisymmetric upper bound solutions are less accurate than the upper bound solutions for plane strain extrusion shown in Figure 2. 


\section{Correspondence between plane strain and axisymmetric extrusion pressures}

Hill suggested in 1948 [6] that reliable estimates for axisymmetric extrusion pressures could be obtained from plane strain slipline field solutions if the area reductions were the same in both cases. Hill's comments were based on agreement between plane strain slipline field results and measured extrusion pressures for axisymmetric metal extrusion, but the hypothesis can now be tested against numerical results. (We shall now adopt the subscripts $p s$ and $a x$ to distinguish, where necessary, between quantities associated with plane strain and axisymmetric deformations.) The plane strain slipline field extrusion pressures from Figure 2 have been replotted in Figure 4, but this time against $\frac{1}{2} \ln \left|1 /\left(1-r_{p s}\right)\right|$, for comparison with axisymmetric extrusion pressures for equal area reductions. The slipline field results are seen to provide much better predictions for the axisymmetric extrusion pressures than the upper bound solutions. Furthermore, a similar correspondence can be found for rough-walled dies, as we shall now demonstrate.

\section{Perfectly rough dies}

\section{Plane strain extrusion}

At a perfectly rough wall, the wall shear stress is equal to the material's shear yield stress. The flow patterns for plane strain extrusion through perfectly rough, square-entry dies show less variation with reduction than those for perfectly smooth dies. A single slipline field solution, given by Hill [6], covers reductions up to $r=0.925$, and a similar solution, due to Johnson [7], covers the range $0.925 \leq r \leq 0.980$. Figure $\mathbf{5}$ shows the deforming zone, static zone and velocity discontinuity in Hill's solution, illustrated for a $60 \%$ thickness reduction. Comparison with Figure 1 reveals that the deforming zone is generally much larger for perfectly rough dies. In the discussion that follows, we shall describe the distance between the die face and the position where the deforming zone boundary meets the container wall as the deforming zone length $d$ (made dimensionless by dividing by the initial half-thickness); this is indicated in Figure 5. The perfectly rough die has a large static zone on the die face, with a curved boundary that coincides with a strong velocity discontinuity. In this case, the deforming zone length is equal to the static zone length, but this is not always true for smooth or partly rough dies.

The extrusion pressure for a rough-walled die depends on the total length of material upstream from

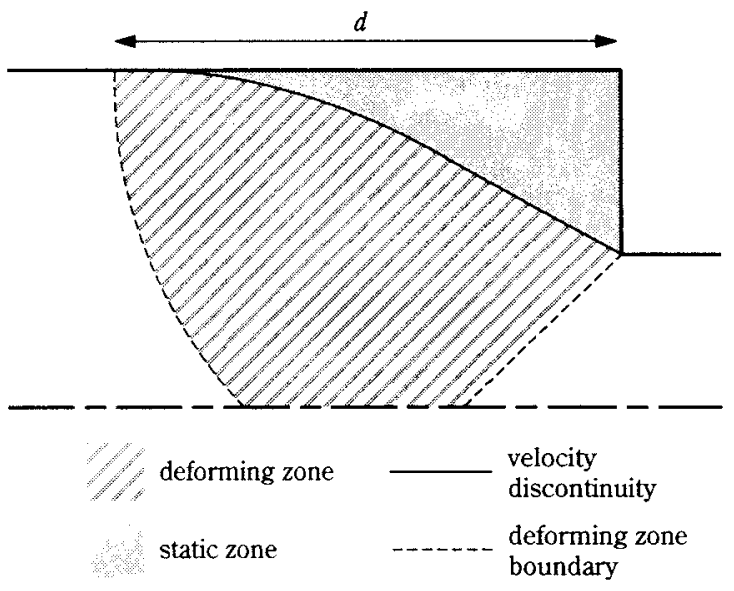

Fig. 5 Deforming zone, static zone and velocity discontinuity for plane strain extrusion through a perfectly rough, squareentry die; from slipline field solution (illustrated for $r=0.6$ ).

the die face, which we term the upstream length $L$ (again relative to the initial half-thickness), and so extrusion pressure results need to be reported more carefully than for smooth-walled dies. The extrusion pressure also depends on the length of material downstream from the die face, but we shall assume that this is zero; the correction for a non-zero downstream length is straightforward. Extrusion pressures obtained from slipline field solutions are often reported as nominal extrusion pressures $P_{n o m}$, which correspond to the energy dissipation associated with the deforming zone only, and do not take into account the energy dissipation due to rigid sliding against the rough container wall. The nominal extrusion pressures for plane strain extrusion through perfectly rough, square-entry dies are plotted against $\ln [1 /(1-r)]$ in Figure 6. These results, calculated from Hill's and Johnson's solutions, are accurately described by the equation

$$
\frac{P_{n o m}}{\sqrt{3} k}=0.01845 \xi^{3}-0.1314 \xi^{2}+1.638 \xi+0.8248
$$

To determine the actual extrusion pressure for a given upstream length (and zero downstream length), an additional term must be added to include the effect of rigid sliding in the container:

$$
P=P_{n o m}+(L-d) k
$$

This correction involves the deforming zone length $d$, which can also be obtained from the slipline field solutions, and is plotted in Figure 6 against the righthand ordinate axis. The deforming zone length does not vary substantially with reduction, being slightly less than 1.5 times the initial half-thickness in all 


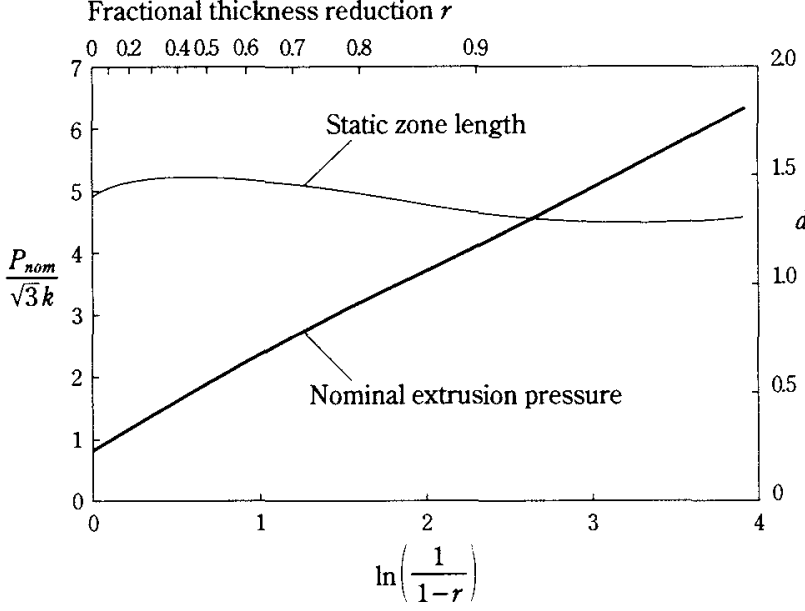

Fig. 6 Nominal extrusion pressures and deforming zone lengths for plane strain extrusion through perfectly rough, squareentry dies; from slipline field solutions.

cases. A suitable correlation describing these results can be constructed from two polynomial arcs:

$$
\begin{array}{rlrl}
d & =1.42+0.310 \xi-0.377 \xi^{2}+0.142 \xi^{3}-0.0192 \xi^{4} & r \leq 0.925 \\
& =1.41-0.0789 \xi+0.0135 \xi^{2} & r \geq 0.925
\end{array}
$$

\section{Axisymmetric extrusion}

A disadvantage with reporting extrusion pressures as nominal values is that these correspond to slightly different upstream lengths depending on reduction and wall friction (and may not correspond to a real extrusion pressure at all if the furthest deforming material from the die face is not located at the container wall). Furthermore, it is difficult to determine the deforming zone length precisely from numerical solutions, which are usually the only accurate solutions available for axisymmetric extrusion. An alternative is to quote extrusion pressures based on a standard upstream length $L^{*}$, and we shall denote the resulting standard extrusion pressure by $P^{*}$. The finite element results plotted in Figure 7 are standard extrusion pressures taken from reference [12] for axisymmetric extrusion through perfectly rough, square-entry dies. In this case, $L^{*}$ was chosen to be 1.5 initial radii from the die face. This is approximately equal to the deforming zone length, and so the contribution to the extrusion pressure from rigid sliding in the container is small. The finite element results are represented well by the straight line fit

$$
\frac{P^{*}}{\sqrt{3} k}=2.41 \xi+1.79
$$

\section{Correspondence between plane strain and axisymmetric extrusion pressures}

Kudo's upper bound solutions for axisymmetric ex-

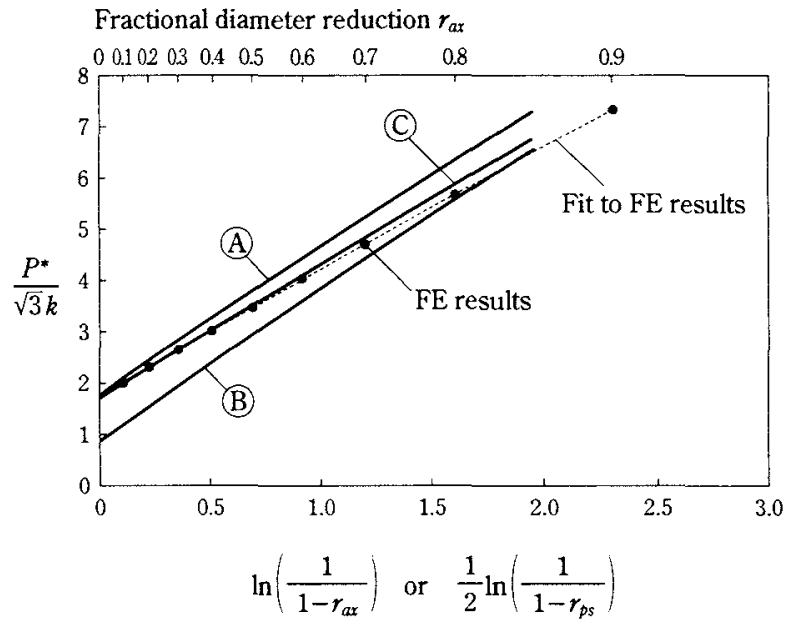

Fig. 7 Standard extrusion pressures for axisymmetric extrusion through perfectly rough, square-entry dies.

trusion [13] can also be applied to perfectly rough, square-entry dies, but as before only provide a good approximation to the finite element results when the reduction is small [12]. A close correspondence between plane strain and axisymmetric results for equal area reductions is therefore valuable. For roughwalled dies, it is necessary to choose an appropriate upstream length to use when evaluating the plane strain extrusion pressures. This was not required for the smooth-wall calculation.

When the upstream length is large, the plane strain value should approach twice the axisymmetric value to account for the different surface area to crosssectional area ratio in the container in the two cases. Any rule relating the plane strain and axisymmetric upstream lengths must reproduce this behaviour. The simplest hypothesis is to make the plane strain upstream length exactly equal to twice the axisymmetric value, even when the upstream length is small. Line $A$ in Figure 7, calculated from equation (8), is based on this hypothesis, with $L_{p s}$ equal to 3 , twice the $L^{*}$ value used when plotting the axisymmetric results. There is good agreement between plane strain and axisymmetric extrusion pressures at small reductions, but the plane strain values are significantly greater than the axisymmetric values at large reductions.

An alternative rule for determining $L_{p s}$, which also produces the correct behaviour when the upstream length is large, is to assume that the difference $(L-d)$ in the plane strain case is twice that in the axisymmetric case. This is equivalent to the assumption that the nominal extrusion pressures for equal area reductions are similar, which was found to work well for smoothwalled dies. We must now estimate the axisymmetric 
deforming zone length, and the two most obvious possibilities are that it is approximately equal to (i) the plane strain deforming zone length for an equal area reduction, or (ii) the plane strain deforming zone length for an equal linear reduction. In fact, because $d_{p s}$ does not vary greatly with reduction, both choices produce similar results. Line B in Figure 7 is based on choice (i), and the plane strain extrusion pressures are found to be in good agreement with the axisymmetric extrusion pressures at large reductions, but significantly less than the axisymmetric values at small reductions

If the nominal extrusion pressures for equal area reductions are indeed similar, then we can deduce that $d_{a x}$ is approximately equal to $d_{p s}$ at large reductions, but is less than $d_{p s}$ at small reductions. The velocity fields obtained from finite element calculations support this conclusion [12], which provides some justification for the following method for estimating the axisymmetric deforming zone length. Since the linear reduction for an axisymmetric extrusion is smaller than for a plane strain extrusion with an equal area reduction, the axisymmetric deforming zone length can be estimated from the plane strain deforming zone length by scaling according to the ratio of the linear reductions:

$$
\frac{d_{a x}}{d_{p s}} \approx \frac{r_{a x}}{r_{p s}}=\frac{1}{1+\sqrt{1-r_{p s}}}
$$

The axisymmetric standard extrusion pressure is then estimated from

$$
P_{a x}^{*} \approx P_{n o m, p s}+2\left(L_{a x}^{*}-\frac{d_{p s}}{1+\sqrt{1-r_{p s}}}\right) k
$$

where the plane strain quantities all correspond to an equal area reduction. Line $C$ in Figure 7 is calculated from this equation, and provides good estimates for the axisymmetric extrusion pressures at all reductions.

\section{Conclusions}

Nominal extrusion pressures appear to be similar for plane strain and axisymmetric extrusions with equal area reductions, for both perfectly smooth and perfectly rough square-entry dies. Since plane strain slipline field extrusion pressures are widely reported, this fact allows axisymmetric extrusion pressures to be rapidly estimated. Even where plane strain extrusion pressures must be specially calculated from slipline field solutions, these calculations are much quicker than a finite element analysis for the axisymmetric problem. When the walls are rough, the need to determine the axisymmetric deforming zone length complicates matters, but an empirical rule for estimating this quantity for perfectly rough, square-entry dies has been described.

This method for estimating axisymmetric extrusion pressures could prove useful in situations where forming loads must be determined quickly and without extreme precision, and where no additional information regarding the stress and velocity fields is required. The method is expected to produce good results for dies with intermediate Tresca boundary conditions, in addition to the perfectly smooth and perfectly rough cases. Furthermore, plane strain slipline field solutions exist for many more complicated extrusion problems. For example, different wall shear stress values can be accommodated on the die face and container wall, and the die geometry itself can also be quite different; solutions are available for tapered and curved die entries, and for unsymmetrical extrusion. Agreement between nominal extrusion pressures for plane strain and axisymmetric (or, in the unsymmetrical case, three-dimensional) extrusions with equal area reductions might also exist in these situations. Such agreement is purely empirical, however, and can only be established by further comparisons between plane strain slipline field results, and numerically or experimentally derived axisymmetric extrusion pressures.

\section{Nomenclature}

\section{Roman}

a Length parameter in upper bound solutions, relative to initial half-thickness

$d$ Static zone length, relative to initial half-thickness or radius

$k \quad$ Shear yield stress

$L \quad$ Upstream length, relative to initial half-thickness or radius

$L^{*} \quad$ Standard upstream length

$P \quad$ Extrusion pressure

$P^{*} \quad$ Standard extrusion pressure, based on upstream length $L^{*}$

$P_{n o m}$ Nominal extrusion pressure

$r \quad$ Linear reduction

\section{Greek}

$\xi$ Logarithm of the thickness or diameter ratio, $\xi=\ln |1 /(1-r)|$

\section{General subscripts}

ax Quantity associated with axisymmetric extrusion

ps Quantity associated with plane strain extrusion 


\section{References}

1) Oliver, D.R. and Whiskens, M.: "The effect of absolute pressure on the processing parameters of ceramic pastes", British Ceramic Proceedings, no. 55 (1996), 87-97.

2) Chakrabarty, J.: "Theory of Plasticity", McGraw-Hill Book Company, New York, U.S.A. (1987).

3) Horrobin, D.J. and Nedderman, R.M.: "Die entry pressure drops in paste extrusion", Chemical Engineering Science, 53 (1998), 3215-3225.

4) Hill, R.: "The Mathematical Theory of Plasticity", Clarendon Press, Oxford, U.K. (1950).

5) Johnson, W.; Sowerby, R. and Venter, R.D.: "Plane-strain Slip-line fields for Metal-Deformation Processes: a Source Book and Bibliography", Pergamon Press, Oxford, U.K. (1982).

6) Hill, R.: "A theoretical analysis of the stresses and strains in extrusion and piercing", Journal of the Iron and Steel Institute, 158 (1948), 177-185.

7) Johnson, W.: "Extrusion through square dies of large reduction", Journal of the Mechanics and Physics of Solids, 4 (1956), 191-198.

8) Green, W.A.: "Extrusion through smooth square dies of medium reduction", Journal of the Mechanics and Physics of Solids, 10 (1962), 225-233.

9) Hill, R.: "On the state of stress in a plastic-rigid body at the yield point", Philosophical Magazine, 42 (1951), 868-875.

10) Kudo, H.: "An upper-bound approach to plane-strain forging and extrusion-I", International Journal of Mechanical Sciences, 1 (1960), 57-83.

11) Kudo, H.: "An upper-bound approach to plane-strain forging and extrusion-II", International Journal of Mechanical Sciences, 1 (1960), 229-252.

12) Horrobin, D.J.: "Theoretical Aspects of Paste Extrusion", Ph.D. Dissertation, University of Cambridge, Cambridge, U.K. (1999).

13) Kudo, H.: "Some analytical and experimental studies of axi-symmetric cold forging and extrusion-l", International Journal of Mechanical Sciences, 2 (1960), 102-127.

\section{Author's short biography}
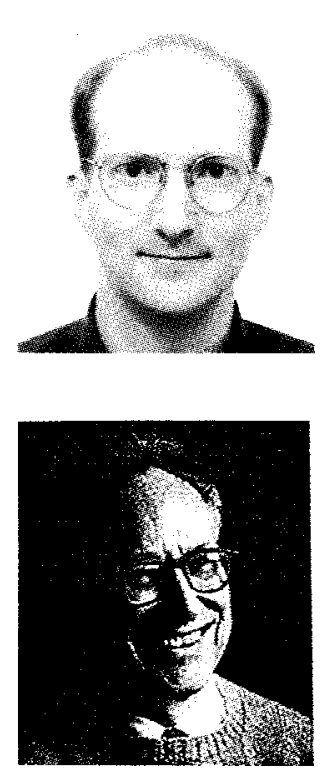

\section{D.J. Horrobin}

D.J. Horrobin studied Chemical Engineering as an undergraduate at Queens' College, Cambridge, and has recently completed a $\mathrm{Ph} . \mathrm{D}$. in the Department of Chemical Engineering at Cambridge University. His Ph.D. dissertation was concerned with modelling the flow of perfectly plastic materials through extrusion dies, with particular reference to the extrusion of soft solid pastes.

In August 1999 he took up a position as a Research Fellow in the Department of Mathematics and Statistics at The University of Melbourne, Australia.

\section{R.M. Nedderman}

R.M. Nedderman has been a lecturer in the Department of Chemical Engineering at Cambridge University since 1960 . He was appointed Ely Fellow of Trinity College, Cambridge in 1981, and is due to retire in July 1999.

His research and teaching has been mainly concerned with Fluid Mechanics and Granular Materials. Up to about 1975, he worked on the Two-Phase Flow of gas/liquid mixtures. Since that date, he has concentrated on the prediction and measurement of stress and velocity distributions in granular materials discharging from hoppers. His recent involvement with the study of the extrusion of plastics results from the similarity between the governing equations for plastic and granular materials. 